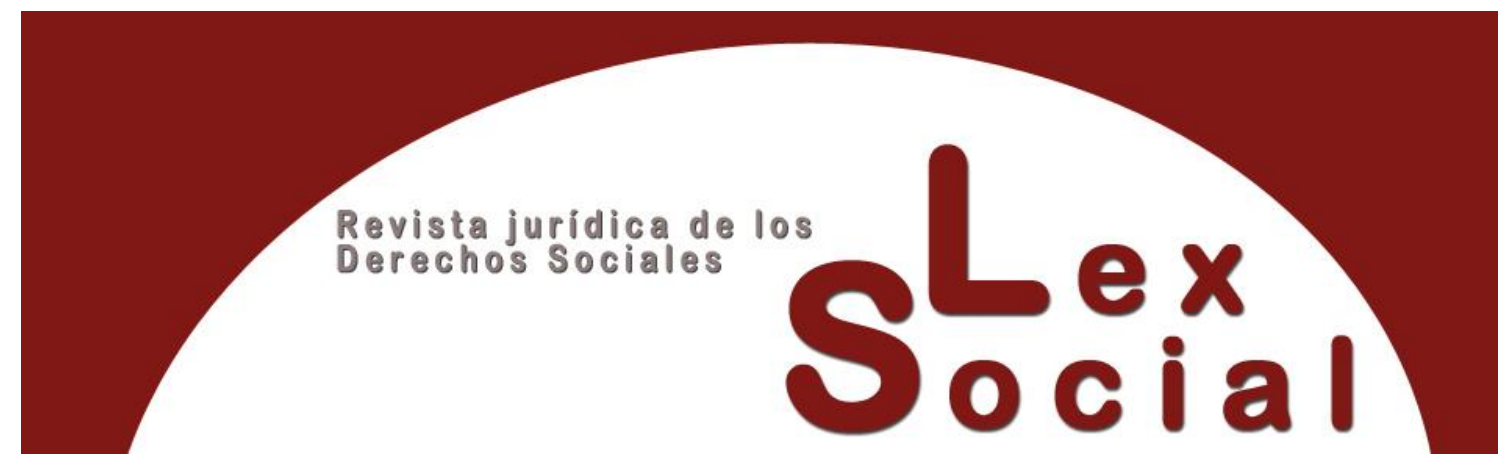

\title{
UWE c. EUROPE: MARIE CURIE S'EN VA-T-EN GUERRE!
}

\section{UWE c. EUROPE: FOR SHE IS A GOOD FELLOW!}

MANUELA BRILLAT

Docteur en droit, Avocate au barreau de Strasbourg https://orcid.org/0000-0003-2602-0002

Cómo citar este trabajo: Brillat, M. (2021). UWE c. Europe: Marie Curie s'en va-t-en guerre! Lex Social: Revista De Derechos Sociales, 11(1), 284-297. https://doi.org/10.46661/lexsocial.5477

\section{RÉSUMÉ}

Le dépôt en 2016 de 15 réclamations collectives par UWE dirigées contre différents États européens n'était pas passé inaperçu. Dans les décisions rendues publiques le 29 juin 2020, le Comité européen des droits sociaux (CEDS) offre une clef de lecture organisée et cohérente indiquant clairement les étapes du raisonnement en matière d'égalité professionnelle. Pour la première fois, le CEDS systématise les obligations à la charge de l'État tant du point de vue de la rémunération que de l'accession des femmes aux postes décisionnels. Cependant, la notion de «progrès mesurables » pourtant essentielle au raisonnement en matière de droits sociaux a un poids hétérogène en fonction de l'aspect analysé. À ne pas l'appliquer rigoureusement, le CEDS risque de donner le flan à ceux qui défendent encore l'injusticiabilité des droits sociaux comme caractéristique intrinsèque.

MoTS-CLÉS: discrimination, égalité, sexe, rémunération, entreprises.

\section{AbStract}

The registration in 2016 of 15 collective complaints by UWE against various European states had not gone unnoticed. In the decisions made public on June 29, 2020, the European Committee of Social Rights (ECSR) offers an organized and coherent reading key, clearly indicating the stages of reasoning in the field of professional equality. For the first time, the ECSR systematizes a list of the obligations on the state both in terms of 
remuneration and women's access to decision-making positions. However, the notion of "measurable progress", which is essential to the reasoning on social rights, has a heterogeneous weight depending on the aspect analyzed. If it is not rigorously applied, the ECSR risks giving a wide berth to those who still defend the unjusticiability of social rights as an intrinsic characteristic.

KEYWORDS: discrimination, equality, sex, compensation, company.

\section{TABLE DE MATIERES}

I. Introduction - Contexte, paramètre et enjeux de la saisine du CEDS.

1. L'égalité salariale : un enjeu de pauvreté et d'exclusion sociale

2. Un vadémécum du raisonnement en matière d'égalité professionnelle

2.1 Le principe "À travail égal, salaire égal »

2.2 La promotion du principe «à travail égal, salaire égal »

2.3 L'accession des femmes aux postes décisionnels

II. De quoi souffre l'Europe?

1. Un manque de transparence salariale : le non-respect de l'obligation positive à la charge de l'État

2. L'absence de progrès mesurables dans l'écart de rémunération entre hommes et femmes

III Ombre et lumière des décisions UWE

1. La lumière (tamisée)

1.1. Le recours ineffectif comme élément de la discrimination

1.2. Une interprétation large de la notion de recours effectif

2. L'ombre : un raisonnement confus en matière de représentation équilibrée des femmes dans les postes décisionnels au sein des entreprises privées

2.1. Une obligation de résultat implicite

2.2. L'exigence non assumée d'une discrimination positive

2.3. Une utilisation ambivalente du concept de "progrès mesurables » IV Conclusion

Bibliographie

\section{Introduction - Contexte, paramètre et enjeux de la saisine du CEDS.}

UWE avait décidé de frapper fort. Le dépôt en 2016 de 15 réclamations collectives dirigées contre différents États européens n'était pas passé inaperçu ${ }^{1}$. L'issue de cette procédure devant le Comité européen des droits sociaux (CEDS) était attendue et elle est

\footnotetext{
${ }^{1}$ Il s'agit des réclamations n ${ }^{\circ} 124$ à 138 contre : la Belgique, la Bulgarie, la Croatie, Chypre, la République tchèque, la Finlande, la France, la Grèce, l'Irlande, l'Italie, les Pays-Bas, la Norvège, le Portugal, la Slovénie et la Suède. Toutes ces décisions contiennent les mêmes développements relatifs aux principes appliqués par le CEDS. Lorsqu'il sera question de les expliciter dans cet article, la décision contre la Belgique, première par ordre alphabétique, servira de référence pour les notes en bas de page.
} 
arrivée à son terme par les décisions adoptées le 6 décembre 2019 et rendues publiques le 29 juin dernier.

Le Groupe européen de femmes diplômées des universités (UWE) est une organisation non gouvernementale fondée en 1909 qui a son siège à Genève en Suisse. Elle comprend un certain nombre de sections nationales au sein de différents États européens. Ses membres sont exclusivement des femmes ayant au moins trois années d'études universitaires derrière elles. Parmi ses membres illustres, figurent plusieurs prix Nobel (dont Marie Curie) et des femmes politiques (dont Mary Robinson). Cette organisation a été à l'origine et est encore de tous les combats relatifs à l'égalité homme-femme, notamment en matière d'accès à l'éducation.

Qui mieux que UWE pour défier le statut quo européen en matière d'égalité salariale devant le CEDS ? La stratégie était claire : déposer une réclamation collective au nom d'UWE et de l'antenne nationale correspondante à l'État défendeur contre tout État Partie à la Charte ayant ratifié la procédure de réclamation collective : la Belgique, la Bulgarie, la Croatie, Chypre, la République tchèque, la France, l'Irlande, le Portugal, la Finlande, la Grèce, l'Italie, les Pays-Bas, la Norvège, la Slovénie et la Suède. Les réclamations avaient toutes déjà été déclarées recevables par le CEDS à qui il ne restait plus qu'à se prononcer sur leur bien-fondé. Élément assez rare pour être souligné : la Commission européenne est intervenue à la procédure pour présenter des observations sur l'état du droit de l'Union européenne en la matière ${ }^{2}$. Ainsi, c'est bien une analyse européenne très globale que le CEDS était invité à effectuer, et c'est bien ensemble que ses décisions doivent être analysées pour révéler la situation européenne en matière d'égalité entre homme et femme sur le marché du travail. Cet aspect se vérifie, d'ailleurs, à la lecture des réclamations qui ont toutes été signées par la même avocate et qui font état d'un constat global partagé avant de préciser les éléments relatifs à chacun des pays concernés.

Le paramètre du contrôle effectué est bien sûr le texte de la Charte sociale (révisée) (CSER) et, plus particulièrement, ses articles $4 \S 3$ (droit à une rémunération équitable), $20 \mathrm{C}$ (droit à l'égalité des chances et de traitement en matière d'emploi et de profession, sans discrimination fondée sur le sexe) et $20 \mathrm{D}$ (droit à l'égalité des chances et de traitement en matière d'évolution de carrière) ${ }^{3}$. Le CEDS explique clairement qu'il utilise, dans son analyse, la technique de l'interprétation évolutive et de l'interprétation globale du texte dont il assure le respect ${ }^{4}$.

\section{L'égalité salariale : un enjeu de pauvreté et d'exclusion sociale}

Le caractère raisonnable d'une rémunération n'est pas uniquement une question mathématique. Elle comporte une dimension sociale importante puisqu'elle est la clef de

\footnotetext{
${ }^{2}$ Le droit de l'Union reconnaît l'égalité entre homme et femme tant dans le droit primaire que dans le droit dérivé, notamment à travers la directive 2006/54/CE relative à la mise en œuvre du principe de l'égalité des chances et de l'égalité de traitement entre hommes et femmes en matière d'emploi et de travail.

${ }^{3}$ Le Comité exclut l'article $\mathrm{E}$ du paramètre considérant que l'interdiction de la discrimination est incluse dans le champ d'application des deux articles (chiffrés) visés par les réclamations.

${ }^{4}$ Voir par exemple: Groupe européen des femmes diplômées des universités (UWE) c. Belgique, réclamation 124/2016, décision sur le bien-fondé adoptée le 6 décembre 2019, §113.
} 
la sortie de la pauvreté et de l'exclusion sociale ${ }^{5}$. C'est bien dans ce cadre que s'inscrit le principe de l'égalité de rémunération entre homme et femme ${ }^{6}$. Ce principe n'est cependant pas le seul évaluateur du respect de l'égalité des sexes en matière professionnelle et de travail : la dimension plus substantielle de l'égalité entre alors en jeu pour assurer un accès à l'emploi, à l'orientation professionnelle et à l'évolution de carrière dénué de toute approche discriminatoire ${ }^{7}$.

À cet égard, le CEDS précise des éléments de définition et de vocabulaire qui viennent s'ajouter aux mots de la discrimination : à la discrimination directe, indirecte, systémique et aggravée, viennent s'ajouter la ségrégation sectorielle, professionnelle ou verticale ${ }^{8}$. Le terme même de «ségrégation» est bien la marque du lien entre, d'une part, discrimination entre les sexes en matière d'emploi et de travail et, d'autre part, l'exclusion sociale qui peut en découler. Le champ lexical du CEDS s'enrichit pour traiter de ces réclamations collectives et son extension n'est pas neutre : elle rappelle l'importance vitale de l'égale dignité entre les êtres humains proclamée par la Déclaration Universelle des Droits de l'Homme.

Le CEDS ne se contente cependant pas, dans ces décisions, de la sphère des principes et des valeurs. Il décortique le droit à l'égalité entre homme et femme en matière professionnelle et d'emploi et offre un vadémécum précieux sur le raisonnement juridique à suivre pour évaluer la situation générale. Là où le droit national et le droit de l'Union apportent des éléments pour l'analyse individuelle d'une affaire (en particulier l'épineuse question de la charge et du mode de preuve ${ }^{9}$ ), le CEDS donne des détails importants sur l'analyse générale.

\section{Un vadémécum du raisonnement en matière d'égalité professionnelle}

Les décisions UWE sont l'occasion pour le CEDS de faire de la pédagogie en matière de raisonnement et d'analyse. Pour ce faire, il regroupe les différents griefs soulevés par l'organisation réclamante en trois catégories distinctes pour construire une grille d'analyse de l'égalité entre homme et femme en matière d'emploi et professionnelle. Il offre ainsi un vadémécum du raisonnement en matière d'égalité, qui ne manquera pas de fonder ses décisions à venir. Il constitue également un outil intéressant à disposition des entreprises et des salarié(e)s pour pouvoir porter un regard large sur leur situation.

Sur le fond, le CEDS reprend des éléments qui apparaissaient déjà dans des conclusions ; toutefois, il développe, pour la première fois, l'ensemble de son raisonnement dans le cadre de ces quinze décisions inédites qu'il saupoudre d'une précision qui lui est classique : face à des obligations complexes à mettre en œuvre, l'État doit atteindre les objectifs « à une échéance raisonnable, au prix de progrès mesurables, en utilisant au

\footnotetext{
5 Voir par exemple: Groupe européen des femmes diplômées des universités (UWE) c. Belgique, réclamation 124/2016, décision sur le bien-fondé adoptée le 6 décembre 2019, §105.

${ }^{6}$ Ibid., $\$ 106$.

${ }^{7}$ Ibid., $\$ 107$.

${ }^{8}$ Ibid., $\$ 112$

${ }^{9}$ Voir pour le détail : BRILLAT, M. Le principe de non-discrimination à l'épreuve des rapports entre les droits européens ; édité par LGDJ, 2015, 471 p.
} 
mieux les ressources qu'ils peuvent mobiliser ${ }^{10}$. Partant, le fait que des progrès restent encore à accomplir n'est pas nécessairement une indication d'une violation de la CSER (Belgique).

\subsection{Le principe « À travail égal, salaire égal »}

La première étape de l'analyse consiste à examiner la réalité du principe : « à travail égal, salaire égal ». Le CEDS indique que les obligations des États en la matière reposent sur quatre fondements : la reconnaissance de ce principe par la loi (au sens strict du terme ${ }^{11}$ ); l'existence de voies de recours effectives pour en sanctionner le non-respect; le respect du principe de transparence dans la rémunération et l'établissement de méthodes de comparaison; l'existence d'organes efficaces de promotion de l'égalité. Dans chaque décision, le CEDS se prononce ainsi sur ces quatre éléments et le constat de (non)conformité à la CSER peut ne valoir que pour l'un d'eux. Par ailleurs, il rappelle que la notion de salaire couvre non seulement le salaire de base mais aussi tous les avantages directs et indirects, en espèces ou en nature, versés par l'employeur en raison de l'emploi ${ }^{12}$. Enfin, l'inscription du principe dans la Constitution ne suffit pas ${ }^{13}$; il doit être expressément reconnu par la loi et mis en œuvre. Le CEDS n'a constaté aucune violation de ce point de vue.

L'analyse faite de ce principe est donc large puisqu'il va jusqu'à inclure l'obligation de mettre en place un ou des organes de promotion de l'égalité, typiquement les Ombudsperson ou Défenseurs des Droits rendus obligatoires par la législation de l'Union. Pour s'assurer de l'efficacité de cet organe, le CEDS a établi un certain nombre de critères : son indépendance, ses ressources, le contenu de son mandant qui doit contenir tant l'aide aux victimes à l'engagement de poursuites que l'adoption de décisions faisant autorité $^{14}$. L'analyse du CEDS est ainsi globale. Ainsi, le fait que cet organe «travaille dans un contexte d'hostilité politique $\gg^{15}$ (Bulgarie) est un élément qui interfère nécessairement avec son efficacité.

\subsection{La promotion du principe « à travail égal, salaire égal »}

La deuxième étape du raisonnement consiste à intégrer l'analyse de la promotion du principe «à travail égal, salaire égal ». Cet aspect, qui peut sembler peu juridique, se décline, d'après le CEDS, par deux obligations précises : l'obligation de collecter des statistiques standardisées pour mesurer l'écart de rémunération; l'obligation d'adopter des mesures de promotion de l'égalité des chances par l'intégration de la dimension de genre. Cette approche insiste sur la dimension collective du problème de l'inégalité de rémunération entre homme et femme et la nécessaire attention de l'État qui doit se donner

\footnotetext{
${ }^{10}$ Groupe européen des femmes diplômées des universités (UWE) c. Belgique, réclamation 124/2016, décision sur le bien-fondé adoptée le 6 décembre 2019, \$205 et 220.

${ }^{11}$ Ibid., $\$ 139$.

12 Ibid., $\$ 139$.

${ }^{13}$ Ibid., $\$ 140$

${ }^{14}$ Ibid., $\$ 167$ et suiv.

${ }^{15}$ Groupe européen des femmes diplômées des universités (UWE) c. Bulgarie, réclamation 125/2016, décision sur le bien-fondé adoptée le 6 décembre 2019 Bulgarie, $§ 160$
} 
les moyens d'une politique efficace. L'on se situe donc bien dans une définition matérielle de l'égalité qui vise plus loin que le simple calcul mathématique des rémunérations. Ainsi, après l'intégration d'un vocabulaire de l'égalité matérielle par le mot « ségrégation », le CEDS souligne dans son raisonnement également qu'il compte s'intéresser aux racines de l'inégalité salariale par la mise en avant d'acteurs clés dans l'étude de la situation nationale.

L'État a, notamment, l'obligation de rechercher les causes de l'écart de rémunération ${ }^{16}$ et d'adapter des mesures adéquates ${ }^{17}$ incluant la dimension de genre ${ }^{18}$. Le CEDS donne alors une première liste de mesures qui peuvent être efficaces ${ }^{19}$ : la mise en œuvre de plans d'action pour l'emploi, exiger des entreprises qu'elles adoptent un plan garantissant l'égalité de rémunération, encourager à traiter cette question dans le cadre de conventions collectives, sensibiliser au principe de l'égalité de rémunération.

\subsection{L'accession des femmes aux postes décisionnels}

La troisième étape du raisonnement consiste à examiner la représentation des femmes aux postes décisionnels dans les entreprises privées. Le CEDS considère que cet aspect fait partie intégrante de l'obligation des États de promouvoir l'égalité des chances et de traitement dans le domaine de l'évolution de carrière, y compris la promotion, prévue à l'article $20 \mathrm{~d}$. de la CSER. Cet aspect s'inscrit dans la lutte contre la ségrégation verticale sur le marché du travail et peut comporter «l'adoption de mesures législatives contraignantes visant à assurer l'égalité d'accès aux conseils d'administration des entreprises $»^{20}$. La politique de quota est donc pleinement validée par le CEDS.

\section{De quoi souffre l'Europe?}

La lecture des décisions du CEDS offre pour la première fois une vision globale de la situation européenne: de manière rassurante, certains clichés sont respectés ${ }^{21}$; de manière plus rassurante encore, d'autres sont balayés ${ }^{22}$. Plus concrètement, le constat est sans appel : l'Europe souffre d'un grand manque de transparence et présente une lacune certaine dans la promotion de l'égalité des chances entre homme et femme.

\footnotetext{
${ }^{16}$ Groupe européen des femmes diplômées des universités (UWE) c. Belgique, réclamation 124/2016, décision sur le bien-fondé adoptée le 6 décembre 2019, \$203.

${ }^{17}$ Ibid., $\$ 205$

${ }^{18}$ Ibid., $\$ 206$

19 Ibid., $\$ 208$

${ }^{20}$ Ibid., $\$ 218$.

${ }^{21}$ Le CEDS ne constate aucune violation par la Suède qui fait donc office de bon élève de l'égalité.

${ }^{22}$ Les pays du sud ne sont pas moins bien lotis que les autres : le Portugal, l'Italie, Chypre ne comptent qu'un constat de violation. Groupe européen des femmes diplômées des universités (UWE) c. Portugal, réclamation 136/2016, décision sur le bien-fondé adoptée le 5 décembre 2019; Groupe européen des femmes diplômées des universités (UWE) c. Italie, réclamation 133/2016, décision sur le bien-fondé adoptée le 6 décembre 2019; Groupe européen des femmes diplômées des universités (UWE) c. Chypre, réclamation 127/2016, décision sur le bien-fondé adoptée le 5 décembre 2019.
} 


\section{Un manque de transparence salariale : le non-respect de l'obligation positive à la charge de l'État}

Onze États sur quinze présentent des insuffisances notables en matière de transparence salariale. Ces insuffisances sont problématiques car elles freinent la reconnaissance d'une discrimination (et donc sa résolution) en empêchant de raisonner selon les étapes propres au droit antidiscriminatoire.

D'une part, à travers l'exigence de transparence salariale, le CEDS confirme une approche très matérielle de l'égalité, dépassant le simple principe d'égalité devant la loi. Il est établi que « la transparence contribue à identifier les préjugés et la discrimination fondés sur le sexe et facilite la prise de mesures correctives tant par les travailleurs que par les employeurs et leurs organisations, ainsi que par les autorités compétentes ${ }^{23}$. La notion de préjugé est désormais partie intégrante du vocabulaire juridique du droit antidiscriminatoire européen ${ }^{24}$. L'intention discriminatoire n'est donc pas un critère de l'établissement de la discrimination dans la mesure où les préjugés ne reflètent pas uniquement une conscience établie d'une différence de traitement illégale mais également des angles-morts. L'exigence de transparence rejoint donc la définition matérielle de l'égalité franchement défendue par le CEDS depuis le début de ses décisions.

D'autre part, le raisonnement du CEDS en matière de transparence rappelle, si besoin était, l'étape essentielle du raisonnement en matière de discrimination à savoir, la comparaison des situations. Ainsi, les employeurs doivent être tenus d'un rapport régulier sur les salaires et de la production de données ventilées par genre ${ }^{25}$. Le CEDS donne, à cet égard, les facteurs qui doivent être pris en considération pour déterminer si un travail est égal à un autre ${ }^{26}$ et les États doivent s'efforcer de clarifier cette notion pour préserver la sécurité juridique ${ }^{27}$. Le CEDS est précis sur ce que les États doivent faire et ne doivent pas faire en matière de comparaison ${ }^{28}$, conscient que cette étape constitue la porte d'entrée dans le monde de la discrimination : en limitant cette étape, c'est nécessairement les constats de discrimination que l'on limite. En somme, il s'agit de prendre appui sur la notion de « travail à valeur égale » et non celle, trop restrictive, de « travail égal ».

Les écueils des États en la matière sont divers: l'absence de ou la mauvaise reconnaissance législative du principe de la transparence salariale et d'un système de classification (Belgique, Bulgarie, Chypre, Croatie, Italie, Pays-Bas, Slovénie), une

\footnotetext{
${ }^{23}$ Groupe européen des femmes diplômées des universités (UWE) c. Belgique, réclamation 124/2016, décision sur le bien-fondé adoptée le 6 décembre 2019, 154.

${ }^{24}$ La Cour européenne prend rarement appui sur la notion de préjugé pour constater l'existence d'une discrimination. Sur l'égalité homme-femme, voir : CEDH du 25 juillet 2017, Carvalho Pinto de Sousa Morais c. Portugal, n¹7484/15 ; sur l'orientation sexuelle, voir : CEDH du 20 juin 2017, Bayev et autres c. Russie, $\mathrm{n}^{\circ} 67667 / 09$ et autres; sur la race et l'origine ethnique, voir : CEDH du 6 juillet 2005, Natchova et autres c. Bulgarie, ${ }^{\circ} 43577 / 98$.

${ }^{25}$ Groupe européen des femmes diplômées des universités (UWE) c. Belgique, réclamation 124/2016, décision sur le bien-fondé adoptée le 6 décembre 2019, §155.

${ }^{26}$ Ibid., $\$ 156$.

${ }^{27}$ Idem.

${ }^{28}$ Ibid., $\$ 157$ et 158 .
} 
jurisprudence rare et restrictive dans la définition du travail de valeur égale (Belgique, Bulgarie, Chypre, Croatie, Grèce, Italie, République Tchèque, Slovénie), champ du comparateur indûment limité (Belgique, Bulgarie, Croatie, Irlande, Norvège, République Tchèque, Slovénie), absence de contrôle ou contrôle insuffisant sur les décisions des entreprises en la matière (Belgique, Bulgarie, Chypre, Croatie, Grèce, Irlande), manque d'accès à l'information des victimes potentielles (Bulgarie, Chypre, Croatie, Grèce, Irlande, Italie, Pays-Bas, République Tchèque, Slovénie).

L'ensemble de ces éléments est pris en considération dans le constat de violation ou de non-violation. Encore une fois, il n'est pas demandé à l'État d'être parfait mais d'aller dans le bon sens avec les moyens dont il dispose ${ }^{29}$. Toutefois, la seule restriction indue du comparateur conduit à un constat de violation (Norvège). Au final, seuls quatre États s'en sortent sans constat de violation : la Finlande, la France, le Portugal et la Suède.

\section{L'absence de progrès mesurables dans l'écart de rémunération entre hommes et femmes}

Les constats de violation de la CSER sur ce point démontrent combien le CEDS entre dans le détail pour analyser concrètement la situation de l'État en cause. Il ne se contente pas de l'existence de statistiques étatiques (Grèce, Irlande) mais il s'intéresse aussi à la méthode pour les établir (Finlande, Italie) et à l'efficacité des mesures adoptées sur leur fondement (Bulgarie, Croatie, Finlande, Norvège, Pays-Bas, Portugal). Si l'écart salarial est inférieur à la moyenne européenne (Belgique) et s'il a diminué ces dernières années (Belgique, Chypre, Suède), le CEDS ne constate pas de violation de la CSER en matière de promotion. Il déduit donc de l'évolution de la situation dans un sens positif l'absence de violation de la CSER. En revanche, le fait que l'écart de rémunération se creuse au fil des ans (Croatie, France) ou qu'il n'évolue pas (Finlande, Norvège, Pays-Bas, Portugal, République Tchèque) conduit inévitablement à un constat de violation. Ainsi, l'évolution de l'écart de rémunération est le curseur du CEDS qui ne se contente pas d'un raisonnement mathématique : quel que soit le taux de cet écart, l'État doit sans cesse chercher à le diminuer $\left(\right.$ Slovénie $\left.^{30}\right)$.

L'Italie sort du lot sur ce point : bien que son taux d'écart de rémunération soit le plus faible parmi les quinze États défendeurs, le CEDS pousse l'analyse pour prendre en considération à la fois l'économie informelle et le taux d'activité féminine. Ce contexte particulier appelle alors une analyse des stéréotypes de genre «compromettant les perspectives sociales et de carrière $»^{31}$ des femmes, y compris dans le système éducatif,

\footnotetext{
29 Voir par ex. : la décision contre la Finlande où le constat de non-violation n'empêche pas la reconnaissance de quelques limitations. Groupe européen des femmes diplômées des universités (UWE) c. Finlande, réclamation 129/2016, décision sur le bien-fondé adoptée le 5 décembre 2019, §155.

${ }^{30}$ La décision contre la Slovénie est intéressante sur ce point : l'écart reste nettement inférieur à la moyenne européenne mais n'a cessé d'augmenter ces dernières années, raison pour laquelle le CEDS constate la violation de la CSER. Groupe européen des femmes diplômées des universités (UWE) c. Slovénie, réclamation 137/2016, décision sur le bien-fondé adoptée le 5 décembre 2019.

${ }^{31}$ Groupe européen des femmes diplômées des universités (UWE) c. Italie, réclamation 133/2016, décision sur le bien-fondé adoptée le 6 décembre 2019, §189.
} 
tout comme les mesures politiques de conciliation entre vie privée et vie professionnelle $^{32}$.

L'analyse du CEDS sur ce point est symptomatique du raisonnement en matière de droits sociaux : la notion de "progrès mesurables », sur laquelle le CEDS s'appuie depuis des années $^{33}$, appelle une analyse qui ne peut être mathématique mais contextuelle et poussée. Il ne se contente donc pas du résultat chiffré ; il recherche un panorama plus large de la situation puisque l'ensemble des mesures adoptées par les États ne peuvent que se situer dans un cadre temporel large $\mathrm{e}^{34}$.

\section{Ombre et lumière des décisions UWE}

\section{La lumière (tamisée)}

\subsection{Le recours ineffectif comme élément de la discrimination}

L'un des points intéressants de ces décisions est le poids accordé par le CEDS à l'existence d'un recours effectif. Il explique que « le droit interne doit prévoir des voies de recours adéquates et effectives en cas d'allégation de discrimination salariale ${ }^{35}$. Audelà de l'expression malheureuse de « discrimination salariale » ${ }^{36}$, l'affirmation du CEDS démontre son attention pour l'effectivité des droits dont il assure le respect. Plus encore, la position du CEDS confirme l'indivisibilité des droits puisque l'exigence d'un recours effectif est souvent cantonnée - à tort - aux droits civils et politiques, considérés comme justiciables contrairement aux droits économiques et sociaux dont la dimension plus programmatique est souvent mise en avant. Or il apparaît clairement dans les décisions UWE que le recours effectif est partie intégrante de la définition de l'égalité dans le sens où tout grief de discrimination doit pouvoir rencontrer une voie judiciaire effective. Le CEDS fait ainsi une nouvelle démonstration de l'inadaptation des catégories de droit à la réalité des droits.

\subsection{Une interprétation large de la notion de recours effectif}

La notion de « recours effectif » telle qu'entendue par le CEDS est bien plus large que celle mise en avant par la Cour européenne des droits de l'Homme, par exemple,

\footnotetext{
32 Ibid., $\$ 193$.

${ }^{33}$ Autisme-Europe c. France, réclamation n 13/02, décision sur le bien-fondé adoptée le 4 novembre 2003.

${ }^{34}$ Cet aspect avait été particulièrement développé par le CEDS dans le cadre de décisions relatives à l'adoption de mesures d'austérité par différents pays, en réponse à la crise financière et économique. Voir par exemple : GENOP-DEI et ADEDY c. Grèce, réclamation n ${ }^{\circ} 65 / 2011$, décision sur le bien-fondé adopté le 23 mai 2012.

${ }^{35}$ Groupe européen des femmes diplômées des universités (UWE) c. Belgique, réclamation 124/2016, décision sur le bien-fondé adoptée le 6 décembre 2019, §145.

${ }^{36}$ De plus en plus souvent, l'adjectif qui suit le mot discrimination ne désigne plus le motif de la discrimination mais le domaine dans laquelle elle se déploie. Cet usage imprécis des termes ajoute de la confusion à un lexique de la discrimination déjà bien fourni. En l'espèce il s'agissait d'une discrimination fondée sur le sexe dans le domaine salarial et non d'une « discrimination salariale ». Voir aussi : CEDH du 11 juin 2020, Baldassi et autres c. France, $\mathrm{n}^{\circ}$ 15271/16: la Cour parle de «discrimination économique » alors qu'il s'agit d'une discrimination raciale ou ethnique ayant des conséquences économiques.
} 
puisqu'elle comprend aussi des dimensions du procès équitable à savoir : l'accès au tribunal $^{37}$ (Bulgarie, Croatie, Grèce), des coûts et des délais raisonnables ${ }^{38}$ (Bulgarie, Croatie, Grèce), une indemnisation suffisante pour réparer le préjudice ${ }^{39}$ (Bulgarie, Croatie). Sur ce dernier point, dans le sillage de sa jurisprudence concernant les indemnités de licenciement ${ }^{40}$, le CEDS précise bien que tout plafonnement en la matière serait contraire à la Charte. La charge de la preuve est également incluse dans la notion de « recours effectif» : en matière de discrimination, cette charge doit être partagée entre la personne qui s'estime victime d'une discrimination et la partie défenderesse ${ }^{41}$. Cet élément n'est pas nouveau puisque prévu par les directives de l'Union. Enfin, le CEDS inclut également dans la notion de « recours effectif » l'interdiction de tout licenciement de représailles ${ }^{42}$. Le CEDS exploite donc au maximum la notion de « recours effectif » en allant au-delà des apparences de procès pour donner des directives claires et précises sur les modalités applicables aux litiges relatifs à la discrimination en matière de rémunération.

Partant de ces principes, le CEDS ne constate qu'une violation du droit à un recours effectif en matière de discrimination qu'à l'égard de cinq pays : la Bulgarie, la Croatie, la Finlande, la Grèce et la Slovénie. Ces conclusions interrogent sur plusieurs points.

Tout d'abord, le nombre de constats de violation de transparence en matière de rémunération ne peut être sans conséquence sur le droit à un recours effectif. En effet, la transparence des rémunérations a deux objectifs principaux, à savoir d'un côté fournir des informations aux États sur l'efficacité des politiques menées et, de l'autre, fournir des informations aux potentielles victimes de discrimination afin de les aider à porter leur affaire devant les tribunaux. Ainsi, il est surprenant, et parfois illogique, de constater d'un côté une lacune massive de l'Europe sur le plan de la transparence et de l'autre n'aboutir qu'à cinq constats de violation du droit à un recours effectif. En effet, sans transparence sur les rémunérations, il est particulièrement difficile de mener une bataille judiciaire puisque la preuve de la discrimination peut être difficile à rapporter, même si le partage de la charge de la preuve est réel : le requérant doit toujours prouver l'existence d'une différence de traitement, or comment la prouver sans informations claires et fiables sur les rémunérations ? Ainsi, le CEDS a fait fi des obstacles liés à l'absence de transparence pour plusieurs pays (Belgique, France, Italie ${ }^{43}$, Pays-Bas, Portugal, République tchèque)

\footnotetext{
${ }^{37}$ Groupe européen des femmes diplômées des universités (UWE) c. Belgique, réclamation 124/2016, décision sur le bien-fondé adoptée le 6 décembre 2019, §145

${ }^{38}$ Idem.

${ }^{39}$ Ibid., $\$ 146$.

${ }^{40}$ Finnish Society of Social Rights c. Finlande, réclamation $n^{\circ} 106 / 2014$, décision sur le bien-fondé adoptée le 8 septembre 2016.

${ }^{41}$ Groupe européen des femmes diplômées des universités (UWE) c. Belgique, réclamation 124/2016, décision sur le bien-fondé adoptée le 6 décembre 2019, §146.

${ }^{42}$ Ibid., $\$ 148$.

${ }^{43}$ Rappelons que la responsabilité de l'Italie a été plusieurs fois reconnue par la Cour européenne en raison de délais de procédure déraisonnables, ce que le CEDS ne semble pas prendre en considération. Or il est difficile d'imaginer que les difficultés de délai qui concernent l'ensemble des procédure italiennes épargnent celles relatives aux affaires de discrimination.
} 
et constate dès lors une conformité à la CSER «malgré les obstacles qui subsistent ${ }^{44}$. Or le principe de progrès mesurables ne s'applique pas au recours effectif qui est une obligation de résultat. Le raisonnement du CEDS est donc peu convaincant sur ce point.

Ensuite, parmi les violations, il faut constater la diversité des motifs qui - souvent indiqués comme uniques ou prépondérants - conduisent à un constat de violation. Pour la Finlande, le fait que la loi ne prévoie pas la réintégration en cas de licenciement par représailles suffit à conclure à une violation. Pour la Grèce, c'est principalement le coût des procédures qui est apparu comme de plus en plus prohibitif. Il semblerait donc qu'il y ait une gradation des éléments constitutifs du droit à un recours effectif sans que cela ne soit affirmé en ces termes dans les principes énoncés par le CEDS. Sinon, comment expliquer qu'un seul élément manquant conduise dans un cas à un constat de violation (Finlande) alors que plusieurs obstacles présents (Belgique) non ?

Enfin, parmi les constats de violation figurent aussi les pays pour lesquels les informations sont limitées sur le nombre d'affaires portées devant les tribunaux (Bulgarie, Slovénie). Le raisonnement paraît logique : si aucune information n'existe, cela signifie que l'attention portée à la question de l'effectivité des recours est moindre. Or, un saut logique est effectué à la lecture des constats de non-violation puisque l'existence d'informations pointant à un faible nombre de recours ne conduit pas toujours à une remise en cause de l'action étatique (Belgique, Chypre, Italie, Norvège, Pays-Bas, Portugal, République tchèque). Le faible nombre de cas portés devant les tribunaux devrait pourtant être un indicateur important de l'effectivité du recours dans une Europe où l'écart de rémunération entre homme et femme est réel.

\section{L'ombre : un raisonnement confus en matière de représentation équilibrée des femmes dans les postes décisionnels au sein des entreprises privées}

En se fondant sur des données statistiques, le CEDS relève que l'adoption de mesures législatives contraignantes permet d'augmenter la représentativité des femmes dans les postes décisionnels ${ }^{45}$. L'objectif doit être celui fixé par l'Assemblée Parlementaire du Conseil de l'Europe, à savoir $40 \%$ des postes décisionnels au sein des entreprises privées occupés par des femmes ${ }^{46}$.

Le raisonnement du CEDS sur ce point est particulièrement confus tant au regard de l'obligation qui pèse sur les États que sur l'intensité du contrôle qu'il opère.

\subsection{Une obligation de résultat implicite}

Le CEDS utilise explicitement le curseur des $40 \%$ pour raisonner. Ainsi, les constats de non-violation concernent soit les États qui ont dépassé ce pourcentage (France, Norvège) ou ceux qui sont dans les dix points qui précèdent (Suède, Italie, Belgique, Finlande, Pays-Bas). Le CEDS repêche également deux États grâce à l'analyse des progrès

\footnotetext{
${ }^{44}$ Groupe européen des femmes diplômées des universités (UWE) c. Belgique, réclamation 124/2016, décision sur le bien-fondé adoptée le 6 décembre 2019, §153.

${ }^{45}$ Ibid., \$219.

${ }^{46}$ Résolution 1715(2010)
} 
mesurables (Portugal et Slovénie). Les constats de violation commencent par indiquer que l'objectif de $40 \%$ n'est pas atteint (Bulgarie, Chypre, Croatie, Grèce, Irlande, République Tchèque) et plus ce chiffre est faible, plus il est par nature suspect (Chypre, Grèce, République Tchèque). Par ailleurs, lorsque des mesures ont été adoptées, le CEDS s'attache à déterminer si l'objectif a été atteint $\left(\right.$ Croatie $^{47}$, Grèce $^{48}$, Irlande $\left.^{49}\right)$ et pas seulement s'il existe un progrès vers cet objectif.

\subsection{L'exigence non assumée d'une discrimination positive}

Le CEDS prend également comme curseur l'existence ou l'absence de mesures législatives contraignantes pour les entreprises privées. Ainsi, tous les constats de violation de la CSER concernent des États dans lesquels il n'existe pas de mesures législatives contraignantes (Bulgarie, Chypre, Croatie, Grèce, Irlande, République Tchèque). En revanche, les constats de non-violation reposent principalement sur l'existence de quotas (Belgique, France, Italie, Norvège, Portugal). L'exemple le plus frappant est celui du Portugal qui, bien qu'il présente un pourcentage faible par rapport aux autres constats de non-violation $(24,8 \%)$, a adopté une législation sur les quotas qui lui permet de bénéficier d'une lecture favorable par le CEDS.

Ainsi, sans jamais l'affirmer, le CEDS admet, voire exige, la mise en place d'une discrimination positive en faveur des femmes pour répondre à l'exigence de la CSER. Or jamais le CEDS n'a utilisé ce vocabulaire - ni le concept qui s'y rattache - dans ses décisions précédentes (?). Il parle d'action positive, c'est-à-dire de législations ou actions en faveur de tel ou tel groupe social. Il n'a jamais parlé de discrimination positive laquelle conduit à une discrimination à rebours pour celui qui n'en bénéficie pas. La discrimination positive consiste à compenser un désavantage et la mise en place de quotas a pour particularité d'influencer, non le processus de décision, mais le résultat. Cette mesure est donc la plus contraignante. Or il n'est pas clair, à la lecture des décisions UWE, si une telle obligation de discrimination positive pèse désormais officiellement sur les États au titre de la CSER. Rappelons que la Cour de Justice de l'Union européenne interprète strictement le traitement préférentiel en faveur des femmes ${ }^{50}$ selon une approche de l'égalité plutôt formelle. Quant à la Cour de Strasbourg, elle admet qu'un traitement préférentiel peut être objectivement justifié au regard des exigences de l'article 14 de la $\mathrm{CEDH}^{51}$.

\subsection{Une utilisation ambivalente du concept de « progrès mesurables »}

La mise en place d'une obligation de résultat déguisée conduit à écarter la notion de « progrès mesurables »- pourtant indiquée comme le cœur de l'obligation étatique. Ainsi,

\footnotetext{
47 Groupe européen des femmes diplômées des universités (UWE) c. Belgique, réclamation 124/2016, décision sur le bien-fondé adoptée le 6 décembre 2019, \$209

${ }^{48}$ Ibid., $\$ 236$

${ }^{49}$ Ibid., $\$ 207$

${ }^{50}$ Voir pour un exemple : CJCE du 6 juillet 2000, Katerina Abrahamsson et Leif Anderson c. Elisabeth Fogelqvist, affaire C-407/98.

${ }^{51}$ Voir par exemple : CEDH du 24 janvier 2017, Khamtokhu et Aksenchik c. Russie, n 60367/08 et 961/11, $\S 71$ et suiv.
} 
elle ne fonde pas le raisonnement du CEDS en la matière alors même qu'elle constitue en principe une étape du raisonnement relatif à la restriction aux droits sociaux fondamentaux. En effet, il s'agit d'un raisonnement en miroir à celui bien connu de l'ingérence dans un droit fondamental : il ne s'agit pas de déterminer jusqu'où l'État peut agir en matière de limitation mais jusqu'où il doit agir en matière de mise en œuvre pour être conforme aux obligations de la CSER. Le seul pays pour lequel ce critère a clairement joué est la Slovénie qui, sans législation contraignante et avec un pourcentage bien en dessous des $40 \%$, bénéficie d'un constat de non-violation en raison de l'augmentation des chiffres. Or, pour la plupart des autres pays, cette même augmentation ne suffit pas, à elle seule, si le chiffre ne frôle pas les $40 \%$ et si l'État ne présente aucune mesure contraignante (Bulgarie, Croatie, Irlande, République tchèque).

Ainsi, il ressort de ces éléments que le raisonnement du CEDS n'est pas linéaire ce qui contribue à rendre nébuleuse l'obligation qui repose effectivement sur les États en matière de représentation des femmes aux postes décisionnels des entreprises privées. Cet aspect contraste avec l'utilisation qui est faite du critère des «progrès mesurables » en matière d'égalité des rémunérations entre hommes et femmes expliqué ci-dessus. Il ne s'agit pas là seulement d'une exigence de rigueur juridique mais bien d'une question d'interprétation de l'exigence de "progrès mesurables »: d'un côté, elle constitue une exigence minimale, de l'autre, elle s'apparente davantage à une marge d'appréciation de l'État. Les décisions UWE ne sortent pas de cette ambivalence.

\section{Conclusion.}

L'enjeu était de taille pour le CEDS qui était saisi, pour la première fois, d'une question commune à tous les États soumis à la procédure de réclamation collective. Il était invité à se prononcer sur un aspect qui n'en finit pas d'être actuel : l'égalité homme-femme au sein du milieu professionnel.

Sur la forme, le CEDS offre une clef de lecture organisée et cohérente indiquant clairement les étapes du raisonnement à suivre en la matière. Cet aspect est fondamental pour le travail du juge national, premier juge des droits. Pour la première fois, le CEDS systématise l'analyse des situations étatiques et permet de faire la liste des obligations à la charge de l'État tant du point de vue de la rémunération que de l'accession des femmes aux postes décisionnels. Le cadre ainsi dessiné révèle non seulement le caractère justiciable des droits sociaux mais aussi leur indivisibilité avec les droits civils et politiques. En effet, l'intégration de la notion de « recours effectif » à la définition de la discrimination est un élément fondamental de l'effectivité du principe d'égalité. En outre, le CEDS utilise les outils classiques des raisonnements en matière de droits humains : la technique des obligations positives, en particulier, et celle de la proportionnalité. Ce dernier point souffre cependant d'une certaine confusion dans sa mise en œuvre ce qui rend, à certains égards, les décisions UWE bancales. 
Ainsi, la notion de «progrès mesurables » pourtant essentielle au raisonnement en matière de droits sociaux a un poids hétérogène en fonction de l'aspect analysé : elle est centrale dans le cadre de la promotion de l'égalité salariale mais marginale dans le cadre de la représentation des femmes aux postes décisionnels. Si cet élément peut sembler accessoire, il est en réalité important puisqu'il s'agit d'un autre versant de la proportionnalité : la notion de «progrès mesurables » fait entrer les droits sociaux dans le champ de la mise en œuvre des droits humains. À ne pas l'appliquer rigoureusement, le CEDS risque de donner le flan à ceux qui défendent encore l'injusticiabilité des droits sociaux comme caractéristique intrinsèque : il révèlerait ainsi ne pas avoir l'autorité de gardien de l'état social européen. 